\title{
Sexual Dysfunction and Associated Anxiety and Depression in Female Hemodialysis Patients: A Cross-Sectional Study at Karachi Institute of Kidney Diseases
}

Sadia Yaqoob ${ }^{1}$, Mahjabeen Yaseen ${ }^{2}$, Hassan Abdullah ${ }^{3,4}$, Furqan Ahmad Jarullah ${ }^{1}$, Uzzam Ahmed Khawaja ${ }^{1}$

1. Medicine, Jinnah Sindh Medical University, Karachi, PAK 2. Nephrology, Fazaia Ruth Pfau Medical College, Karachi, PAK 3. Neurology, University of Alabama, Birmingham, USA 4. Medicine, Nishtar Medical University, Multan, PAK

Corresponding author: Hassan Abdullah, dochassanabdullah@gmail.com

\section{Abstract \\ Objectives}

Hemodialysis patients have to combat certain negative effects such as sexual dysfunction, depression, and anxiety. This study aimed to measure the sexual function and identify the relationship between sexual dysfunction, depression, and anxiety in females undergoing hemodialysis.

\section{Methods}

The descriptive, cross-sectional study was conducted at a dialysis unit in November 2019. Fortyeight females were enrolled in the study. Participants were interviewed for sociodemographic, clinical, and biochemical parameters. Sexual function was assessed through the Female Sexual Function Index (FSFI) while depression and anxiety scores were calculated using the hospital anxiety and depression scale (HADS).

\section{Results}

In this study, the mean age of patients was $44.60 \pm 10.27$ years. Median sexual function scores were low across all domains. A maximum possible score of 3.4 was calculated for the satisfaction domain while the minimum score calculated was 0 for arousal, lubrication, and orgasm. $14.6 \%$ and $45.8 \%$ were suffering from borderline abnormal and abnormal depression, respectively. However, $33.3 \%$ and $31.3 \%$ had borderline abnormal and abnormal anxiety, respectively. Pearson's correlation showed a significant negative correlation between age and desire domain $(r=-0.343 ; p<0.05)$ and demonstrated that arousal, lubrication, orgasm, satisfaction, and pain domains were associated with borderline abnormal depression. None of the sexual domains were correlated with anxiety.

Received 07/28/2020

Review began $08 / 23 / 2020$ Review ended 08/23/2020 Published 08/31/2020

\section{() Copyright 2020}

Yaqoob et al. This is an open access article distributed under the terms of the Creative Commons Attribution License CC-BY 4.0., which permits unrestricted use, distribution, and reproduction in any medium, provided the original author and source are credited.

\section{Conclusions}

Sexual dysfunction, depression, and anxiety are highly prevalent in hemodialysis patients. In this study, borderline abnormal depression was independently linked to sexual dysfunction excluding sexual desire. Therefore, healthcare teams should keep up with the progress of their patients and evaluate for psychosexual health so that they can be timely managed.

Categories: Internal Medicine, Urology, Nephrology

Keywords: sexual dysfunction, hemodialysis, anxiety, depression

\section{Introduction}

End-stage renal disease (ESRD) refers to chronic kidney disease that is so severe in which death is likely without renal replacement therapy (dialysis and renal transplantation) [1]. Currently, hemodialysis is trending since patients are falling short of organs to transplant due to the escalating global burden of ESRD [2]. Hemodialysis remains the treatment of choice also because it helps to optimize patients' functional status and increase life expectancy. However, those undergoing dialysis needs to cope up with certain negative effects including loss of libido, anxiety, and depression $[3,4]$.

Sexual dysfunction refers to a lack of sexual interest or willingness [5]. The worldwide incidence of sexual dysfunction in female hemodialysis patients is reported to be $25-64 \%$ [6]. Common symptoms associated with female sexual dysfunction include low sexual desire, diminished vaginal lubrication, pain and discomfort upon intercourse, minimal sense of arousal, and difficulty in achieving orgasm [7]. However, only a small percentage of women seek medical attention since they are hesitant or embarrassed to discuss it. 
Nationwide, the estimated prevalence rates of depression and anxiety in 2015 were $4.4 \%$ and $3.6 \%$, respectively, per WHO, which incremented by 18\% over the period 2005-2015 [9].

Depression is a mood disorder featured by sadness, hopelessness, and loss of interest or pleasure in daily activities. Greater severity of depressive symptoms in dialysis patients is associated with higher risks for hospitalization, economic crisis, and death. Despondency becomes more pronounced when the patient gradually fails to adhere to the prescribed dialysis regimen, diet, and/or medications [10]. On a different note, the American Psychological Association defines anxiety as an emotion characterized by feelings of tension, worried thoughts, and physical changes like increased blood pressure [11]. After each dialysis episode, anxiety hovers over, culminating in tiredness (71\%), itching (55\%), appetite dysregulation (49\%), pain (47\%), insomnia (44\%), and constant agitation (38\%) [12].

Whilst dialysis units continue to expand all over Karachi, fewer studies have been concentrated here. However, rising rates of ESRD and its related morbidity and mortality have prompted us to evaluate the common issues faced by female hemodialysis patients. Can females under hemodialysis still enjoy sexual activity? Are depression and anxiety correlated with sexual dysfunction? Such questions stirred us to conduct our study where we aim not only to tie-up the links between sexual dysfunction, depression, and anxiety, but also gauge sexual functioning using the Female Sexual Function Index (FSFI).

\section{Materials And Methods}

\section{Study setting}

This study was conducted in a tertiary hemodialysis unit, Karachi Institute of Kidney disease, located in the F.B. Area of Karachi.

\section{Subjects, sample size, and sampling technique}

All females receiving hemodialysis during different work shifts on weekdays during November 2019 were approached. After having met the inclusion criteria, they were included in the study.

\section{Study design}

The research approach employed a descriptive, cross-sectional study to investigate sexual dysfunction and associated anxiety and depression in female hemodialysis patients.

\section{Data collection tools}

The objectives of the study were explained to each participant, and after obtaining the consent, subjects were interviewed as per the questions listed in the questionnaire. The questionnaire comprised of three sections: sociodemographic, clinical, and biochemical parameters constituted the first section which included age, number of children, weight, height, BMI, level of education, occupation, menstrual status, residence, cause of renal failure, duration of kidney disease, duration of hemodialysis, number of dialysis per week, a shift of dialysis, duration of each session of dialysis, any other comorbid, Hb levels, erythropoietin, Venofer injections, calcium, phosphorous, albumin, and parathyroid hormone levels.

The second section included the 19-item self-report questionnaire to measure sexual functioning in women - FSFI [13]. Over the past four weeks, six domains such as desire, arousal, lubrication, orgasm, satisfaction, and pain, each having a maximum score of 6 , were assessed to construe the presence of sexual dysfunction if scores equal to or below 26.55 [14].

The third section encompassed the hospital anxiety and depression scale (HADS) questionnaire which is used to extrapolate the degree of depression and anxiety amongst subjects. HADS is a self-administered questionnaire consisting of seven-depression-related and seven-anxiety-related items [15]. For each variable, a score of 0-7 is considered normal; 8-10 is a borderline case, while 11-21 is considered an abnormal case.

\section{Inclusion criteria}

Patients included in the study were aged between 18 and 65 years, living with their husbands for the past one month and had complete medical records.

\section{Exclusion criteria}

Those excluded from the study were unmarried women or widows, women with a major psychiatric disorder, and those who declined to participate in the study.

\section{Method of data analysis}

Descriptive statistics were generated, based on frequencies for categorical data, or means \pm standard 


\section{Cureus}

deviations (SDs) for quantitative data. Pearson's correlation analysis was used to compare categorical variables. A P-value of $<0.05$ was considered statistically significant. All data were analyzed using SPSS Software 25.0 (SPSS, Inc., Chicago, USA).

\section{Ethical consideration}

Ethics approval was obtained from the Ethical Board Review while permission was taken from the institute. Consents and permission to access medical records were taken from all the participants and they were ensured that confidentiality will be granted to them.

\section{Results}

After having met the inclusion criteria, 48 females volunteered to take part in the study. Table 1 exemplifies the demographics and clinical parameters of patients enrolled in the study.

Variable

Patients demographics

Age (years)

BMI $\left(\mathrm{kg} / \mathrm{m}^{2}\right)$ mean $\pm \mathrm{SD}$

Education (n, \%)

Iiliterate

Primary

Secondary

University education

Occupation (n, \%)

Working

Housewives

Menstrual status (n, \%)

Postmenopausal

With menstrual cycle

Residence ( $n$, \%)

Urban

Rura

Duration of hemodialysis (months)

Cause of renal failure $(n, \%)$

Diabetes mellitus

Hypertension

Glomerulonephritis

Renal stone disease

Urinary Infections

Cystic kidney disease

Unknown cause

Other diseases (n, \%)

Hepatitis B

Hepatitis C

Cardiovascular diseases
Study samples $(n=48)$

28-64

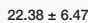

11 (22.9)

$4(8.3)$

$24(50.0)$

$9(18.8)$

2 (4.2)

$46(95.8)$

$29(60.4)$

$19(39.6)$

47 (97.9)

$1(2.1)$

$32.31 \pm 40.61$

$9(18.8)$

$39(81.3)$

0

$3(6.3)$

$3(6.3)$

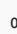

2(4.2)

$1(2.1)$

6 (12.5)

$7(14.6)$ 


\section{Cureus}

$\begin{array}{lc}\text { Shirt }(n, \text { \%) } & 20(41.7) \\ \text { Moming } & 16(33.3) \\ \text { Aftermoon } & 12(25.0) \\ \text { Evening } & \\ \text { Patient clinical characteristics } & 9.72 \pm 1.82 \\ \text { Hb (g/l) } & 8.96 \pm 1.31 \\ \text { Calcium (mmol/) } & 5.87 \pm 1.50 \\ \text { Phosphorous (mmol/) } & 3.55 \pm 0.74 \\ \text { Albumin (g/) } & \end{array}$

TABLE 1: Demographics and clinical parameters of patients

Their mean age was $44.60 \pm 10.27$ while the average duration of hemodialysis was $32.31 \pm 40.61$ months. Most of the patients were postmenopausal (60.4\%) and working as housewives (95.8\%). The majority viewed that the leading causes of renal failure were diabetes (18.8\%) and hypertension (81.3\%).

As for the arousal, lubrication, orgasm, satisfaction, and pain domains, 33 patients (68.8\%) had no sexual activity. Only one patient (2.1\%) accounted for very low or no sexual arousal, two (4.2\%) of them found it extremely difficult or impossible to lubricate, four patients (8.3\%) had difficulty in reaching orgasm, none were very dissatisfied with their overall sex life, and only one patient (2.1\%) reported to have high pain during sexual activity. Median scores enlisted in Table 2 were: desire (1.2), arousal (0), lubrication (0), orgasm (0), satisfaction (3.4), pain (0), and a total score (5.2).

\begin{tabular}{|c|c|c|c|c|}
\hline Domains & Mean \pm SD & Median & $25^{5^{h}}$ percentile & $75^{\text {th }}$ percentile \\
\hline Desire & $1.925 \pm 1.0052$ & 1.2 & 1.2 & 2.85 \\
\hline Arousal & $1.175 \pm 1.8749$ & 0 & 0 & 3 \\
\hline Lubrication & $1.494 \pm 2.3683$ & 0 & 0 & 3.75 \\
\hline Orgasm & $1.467 \pm 2.2812$ & 0 & 0 & 3.9 \\
\hline Satisfaction & $3.658 \pm 1.6357$ & 3.4 & 2.4 & 5.4 \\
\hline Pain & $1.233 \pm 2.0477$ & 0 & 0 & 2 \\
\hline Total score & $10.702 \pm 9.8308$ & 5.2 & 4.25 & 20.075 \\
\hline
\end{tabular}

TABLE 2: The score distribution of each sexual function domain

Patients' HADS scores of anxiety and depression ranged from 0 to 21 . Of all the patients assessed for depression, 19 (39.6\%) were normal, 7 (14.6\%) were borderline abnormal, and 22 (45.8\%) were abnormal. However, the severity of anxiety differed a bit with 17 patients (35.4\%) being normal, 16 (33.3\%) with the borderline abnormal case, and 15 (31.3\%) of being abnormal variety (Table 3).

\begin{tabular}{|c|c|c|c|}
\hline Variables & Normal, $\mathbf{n}(\%)$ & Borderline abnormal, $\mathrm{n}(\%)$ & Abnormal, $\mathbf{n}(\%)$ \\
\hline Depression & $19(39.6)$ & $7(14.6)$ & $22(45.8)$ \\
\hline Anxiety & $17(35.4)$ & 16 (33.3) & $15(31.3)$ \\
\hline
\end{tabular}

TABLE 3: Severity of depression and anxiety in patients undergoing hemodialysis 
Pearson's correlation was run to measure the association of age, depression, and anxiety with various sexual domains (Table 4).

\begin{tabular}{|c|c|c|c|c|c|c|}
\hline & Desire score & Arousal score & Lubrication score & Orgasm score & Satisfaction score & Pain score \\
\hline \multirow{2}{*}{ Age } & $R=-0.343^{*}$ & $R=-0.095$ & $R=-0.089$ & $R=-0.077$ & $R=-0.071$ & $R=-0.149$ \\
\hline & $P=0.017$ & $P=0.521$ & $P=0.550$ & $P=0.601$ & $P=0.631$ & $P=0.311$ \\
\hline \multicolumn{7}{|l|}{ Depression } \\
\hline \multirow{2}{*}{ Normal } & $R=-0.287$ & $R=-0.241$ & $R=-0.061$ & $R=-0.067$ & $\mathrm{R}=0.011$ & $R=-0.087$ \\
\hline & $P=0.233$ & $\mathrm{P}=0.323$ & $\mathrm{P}=0.805$ & $P=0.785$ & $\mathrm{P}=0.964$ & $P=0.723$ \\
\hline \multirow{2}{*}{ Borderline abnormal } & $R=-0.298$ & $R=-0.798^{\star}$ & $R=-0.871^{\star}$ & $R=-0.839^{\star}$ & $R=-0.781^{*}$ & $R=-0.757^{*}$ \\
\hline & $P=0.516$ & $P=0.031$ & $P=0.011$ & $P=0.018$ & $\mathrm{P}=0.038$ & $P=0.049$ \\
\hline \multirow{2}{*}{ Abnormal } & $R=-0.262$ & $R=-0.059$ & $R=-0.248$ & $R=-0.174$ & $\mathrm{R}=-0.068$ & $R=-0.064$ \\
\hline & $P=0.238$ & $P=0.794$ & $P=0.267$ & $P=0.439$ & $P=0.765$ & $P=0.778$ \\
\hline \multicolumn{7}{|l|}{ Anxiety } \\
\hline \multirow{2}{*}{ Normal } & $\mathrm{R}=0.259$ & $R=-0.337$ & $R=-0.227$ & $R=-0.263$ & $\mathrm{R}=-0.360$ & $R=-0.360$ \\
\hline & $P=0.315$ & $P=0.187$ & $P=0.381$ & $P=0.307$ & $P=0.156$ & $P=0.156$ \\
\hline \multirow{2}{*}{ Borderline abnormal } & $R=0.154$ & $R=-0.118$ & $R=-0.070$ & $R=-0.041$ & $\mathrm{R}=0.021$ & $R=-0.109$ \\
\hline & $P=0.569$ & $P=0.665$ & $P=0.797$ & $P=0.880$ & $P=0.939$ & $\mathrm{P}=0.689$ \\
\hline \multirow{2}{*}{ Abnormal } & $R=-0.466$ & $R=-0.359$ & $R=-0.371$ & $R=-0.372$ & $R=-0.248$ & $R=-0.360$ \\
\hline & $P=0.080$ & $P=0.189$ & $P=0.173$ & $P=0.172$ & $P=0.373$ & $P=0.188$ \\
\hline
\end{tabular}

TABLE 4: Pearson's correlation coefficient results of age, depression, and anxiety with sexual function domains in hemodialysis

${ }^{*}$ Correlation is significant at the 0.05 level (two-tailed).

The results revealed a significant negative correlation between age and desire domain $(r=-0.343 ; p<0.05)$. The results also showed a negative relationship between arousal, lubrication, orgasm, satisfaction, and pain domain with borderline abnormal depression which were considered significant. Conversely, sexual domains were not significantly correlated with anxiety.

\section{Discussion}

Even though the renal replacement therapy is suggestive of upgrading the quality of life of those suffering from ESRD, it has seriously hampered the psychosexual life of an individual [16].

Sexual dysfunction is multi-faceted and includes increasing age, menopausal status, depression, and comorbidities like diabetes and hypertension as its major predictors. Literature also shows a discrepancy in the relationship between older age and depression with sexual functioning $[17,18]$. In particular, the results of this study manifested that age had a significant negative correlation with sexual desire, but not with arousal, lubrication, orgasm, satisfaction, or pain. This implies that elderly women experienced a gradual decrease in libido as part of the aging phenomenon than merely due to the disease since the remaining sexual domains were not affected.

Several types of research are inclined towards assessing the degree of depression among hemodialysis patients, while sexual health remained under-recognized [19]. This study was improvised to evaluate the degree of sexual dysfunction in addition to depression and anxiety and find the association between them.

Sexuality was evaluated using a 19-item FSFI over the past four weeks in all six domains: sexual desire, arousal, lubrication, orgasm, satisfaction, and pain. Of all the domains, sexual desire was affected a great deal while none of them reported high levels of dissatisfaction concerning their sexual life. These findings implicit that sexual satisfaction has not just been boosted by increased sexual desire; additive analysis is 
required to evaluate biological, social, and other parameters influencing women's sexual satisfaction. Similar results were also seen in a study conducted by Saglimbene et al. [20] and Weisbord [21]. On account of sexual inactivity, median scores were nil across arousal, orgasm, lubrication, and pain subscales of FSFI, whereas the satisfaction score was still raised. This corroborates with the findings of a study conducted by Nasrallah et al. [22].

As the disease progresses, depression and/or anxiety sneak up and it not only damages an individual's selfesteem but also ruins sexual life [23]. Guven et al. found out that both anxiety and depression were significantly related to sexual dysfunction [24]. Contrary to the results from Esen et al. [5], the present study construed a significant negative correlation between borderline abnormal depression and all sexual domains except sexual desire; meaning borderline abnormal depression scores led to impaired sexual functioning but did not alter libido. Previous studies have failed to correlate the degree of depression with each subset of FSFI. However, anxiety and sexual dysfunction were not inter-related in the current study.

This study was limited in several aspects. First, the sample size was too small. Second, the results of a single-center approach were not sufficient to generalize the findings to the whole population. Third, other parameters impacting sexual life like sociocultural, hormonal, neurovascular, and medical effects were not looked upon. Hence, further evaluation can help to improve substantial findings.

\section{Conclusions}

Patients undergoing hemodialysis not only have to battle the disease but also with subsequent complications arising from it. Depression and anxiety add up to the ordeal. In this study, borderline abnormal depression was independently linked to sexual dysfunction excluding sexual desire. The plan should be outlined to deal with the upcoming challenges, particularly those concerning mental and sexual health. Healthcare teams should keep up with the progress of their patients and evaluate such issues if suspicion arises. Patients should also be encouraged to discuss their health issues frankly with their doctors so that they can be timely evaluated and managed.

\section{Additional Information \\ Disclosures}

Human subjects: Consent was obtained by all participants in this study. Animal subjects: All authors have confirmed that this study did not involve animal subjects or tissue. Conflicts of interest: In compliance with the ICMJE uniform disclosure form, all authors declare the following: Payment/services info: All authors have declared that no financial support was received from any organization for the submitted work. Financial relationships: All authors have declared that they have no financial relationships at present or within the previous three years with any organizations that might have an interest in the submitted work. Other relationships: All authors have declared that there are no other relationships or activities that could appear to have influenced the submitted work.

\section{References}

1. Teles F, Azevedo VF, Miranda CT, Miranda MP, Teixeira M, Elias RM: Depression in hemodialysis patients: the role of dialysis shift. Clinics. 2014, 69:198-202. 10.6061/clinics/2014(03)10

2. Santos PR, Capote JR Jr, Cavalcanti JU, Vieira CB, Rocha AR, Apolônio NA, Oliveira EB: Quality of life among women with sexual dysfunction undergoing hemodialysis: a cross-sectional observational study. Health Qual Life Outcomes. 2012, 10:103. 10.1186/1477-7525-10-103

3. Shafipour V, Alhani F, Kazemnejad A: A survey of the quality of life in patients undergoing hemodialysis and its association with depression, anxiety, and stress. J Nurs Midwifery Sci. 2015, 2:29-35. 10.7508/JNMS.2015.02.004

4. Balaban ÖD, Aydin E, Keyvan A, Yazar MS, Tuna Ö, Devrimci Özgüven H: Psychiatric comorbidity, sexual dysfunction, and quality of life in patients undergoing hemodialysis: a case-control study. Noro Psikiyatr Ars. 2017, 54:137-142. 10.5152/npa.2016.12677

5. Esen B, Kahvecioglu S, Atay AE, et al.: Evaluation of relationship between sexual functions, depression, and quality of life in patients with chronic kidney disease at the predialysis stage. Ren Fail. 2015, 37:262-267. 10.3109/0886022X.2014.990348

6. Gatmiri SM, Bordbar M, Raisi F, Nourian A: Sexual dysfunction in female hemodialysis patients: a crosssectional study in Iran. Acta Med Iran. 2019, 57:244-252. 10.18502/acta.v57i4.1845

7. Karabulutlu EY, Okanli A, Sivrikaya SK: Sexual dysfunction and depression in Turkish female hemodialysis patients. Pakistan J Med Sci. 2011, 27:842-846. 10.12669/pjms.274.488

8. Cengić B, Resić H: Depression in hemodialysis patients. Bosn J Basic Med Sci. 2010, 1:73-78. 10.17305/bjbms.2010.2653

9. Depression and other common mental disorders: global health estimates . (2017). Accessed: August 20, 2020: http://apps.who.int/iris/bitstream/10665/254610/1/WHO-MSD-MER-2017.2-eng.pdf.

10. King-Wing Ma T, Kam-Tao Li P: Depression in dialysis patients. Nephrology. 2016, 21:639-646. 10.1111/nep.12742

11. Anxiety. (2020). Accessed: August 20, 2020: https://www.apa.org/topics/anxiety.

12. Vecchio M, Palmer SC, Tonelli M, Johnson DW, Strippoli GF: Depression and sexual dysfunction in chronic kidney disease: a narrative review of the evidence in areas of significant unmet need. Nephrol Dial Transplant. 2012, 27:3420-3428. 10.1093/ndt/gfs135 
13. Rosen R, Brown C, Heiman J, et al.: The Female Sexual Function Index (FSFI): a multidimensional selfreport instrument for the assessment of female sexual function. J Sex Marital Ther. 2000, 26:191-208. 10.1080/009262300278597

14. Mor MK, Sevick MA, Shields AM, et al.: Sexual function, activity, and satisfaction among women receiving maintenance hemodialysis. Clin J Am Soc Nephrol. 2014, 9:128-134. 10.2215/CJN.05470513

15. Zigmond AS, Snaith RP: The hospital anxiety and depression scale. Acta Psychiatr Scand. 1983, 67:361-370. 10.1111/j.1600-0447.1983.tb09716.x

16. Navaneethan SD, Vecchio M, Johnson DW, et al.: Prevalence and correlates of self-reported sexual dysfunction in CKD: a meta-analysis of observational studies. Am J Kidney Dis. 2010, 56:670-685. 10.1053/j.ajkd.2010.06.016

17. Sabanciogullari S, Taşkın Yılmaz F, Güngör Fİ, Söylemez S, Benli RB: Sexual function in patients with chronic renal failure on hemodialysis and its effects on patients' perception of health and life satisfaction. Sex Disabil. 2015, 33:175-186. 10.1007/s11195-015-9398-4

18. Teuwafeu D, Ashuntantang G, Essi M-J, et al.: Sexual function and correlates in women undergoing maintenance hemodialysis in Cameroon: a multi-centric study. Open Urol Nephrol J. 2016, 9:51-59. 10.2174/1874303X01609010051

19. Aghakhani N, Fattahi Y: Depression in hemodialysis patients. Saudi J Kidney Dis Transplant. 2019, 30:261262 .

20. Saglimbene V, Natale P, Palmer S, et al.: The prevalence and correlates of low sexual functioning in women on hemodialysis: a multinational, cross-sectional study. PLoS One. 2017, 12:e0179511. 10.1371/journal.pone.0179511

21. Weisbord SD: Female sexual dysfunction in ESRD: an underappreciated epidemic? . Clin J Am Soc Nephrol. 2012, 7:881-883. 10.2215/CJN.03870412

22. Nasrallah, Y, Marie R, Eyada M: Female sexual function and depressive symptoms among premenopausal females on hemodialysis. Hum Androl. 2019, 9:34-39. 10.21608/ha.2019.15151.1049

23. Aribi L, Masmoudi R, Ben Houidi A, Charfeddine F, Jarraya F, Hachicha J, Amami O: Sexual disorder in hemodialysis patients. Tunis Med. 2015, 93:79-84.

24. Guven S, Sari F, Inci A, Cetinkaya R: Sexual dysfunction is associated with depression and anxiety in patients with predialytic chronic kidney disease. Eurasian J Med. 2018, 50:75-80. 10.5152/eurasianjmed.2018.17152 